\title{
CrowdTracing: Overcrowding Clustering and Detection System for Social Distancing
}

\author{
Eiman Kanjo, Dario Ortega Anderez, Amna anwar \\ Smart Sensing Lab \\ Department of Computer Science \\ Nottingham Trent University, Nottingham, UK \\ eiman.kanjo@ntu.ac.uk
}

\author{
Ahmad Alshami \\ James William \\ Department of Computer Science Department of Computer Science \\ Southern Arkansas University \\ Magnolia, Arkansas, USA \\ University of Nottingham \\ Nottingham, UK
}

\begin{abstract}
Maintaining social distancing in public spaces plays a pivotal role in decreasing COVID-19 contagion and viral spread. COVID-19 has required many countries around the world to close work places, schools and public spaces. This has prompted policy makers, venue managers and local authorities to investigate practical mitigation strategies using technology to exit the lockdown safely and enable the reopening of cities and public spaces. This paper introduces CrowdTracing, a dynamic overcrowding detection system that encourages social-distancing and triggers an alert to venue, city council or facility managers in a dynamic and privacy-preserving manner. CrowdTracing utilises ubiquitous WiFi probing and density-based clustering techniques which can be performed in real-time to identify commonly crowded areas and assist in the estimation of excess gatherings. The proposed system can also be used to enable discovery of where social distancing rules are not being followed, enabling a rapid response, controlling or slowing down the spread of the virus. A classification recall of 0.85 on an experiment with 1000 simulated scenarios were achieved. This indicates the CrowdTracing system proposed was able to identify 85 out 100 scenarios in which social distancing rules were not being followed.

Index Terms-COVID-19, Ubiquitous Computing, Wireless probe requests, Mobile Sensing, Clustering, People Count, DBSCAN, SOM
\end{abstract}

\section{INTRODUCTION}

The COVID-19 outbreak has changed the way people navigate the world. A large proportion of the population now work or study from home and purchase items over the internet. The situation has the potential to cause significant harm to local economies, affecting high streets, shopping centres, and educational facilities. As local authorities open their venues and city centres and communities begin to return to work and school, the focus has shifted from avoiding contact completely to ensuring social distancing is followed to prevent further outbreaks of COVID-19 [26] [4].

Many policy makers and local authorities are rapidly developing strategies to recover and reopen cities and high streets. In particular they are looking for robust technologies to monitor public spaces and disperse overcrowding. For example, the Federal Office of Public Health (FOPH) has requested analyses from Swisscom to check whether the measures to protect against COVID infections are being observed. To make crowds of people visible, the Swisscom Mobility Insight platform

Identify applicable funding agency here. If none, delete this.

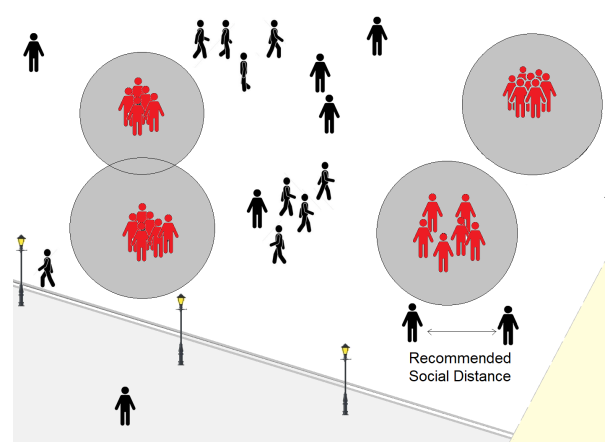

Fig. 1: Illustration of gatherings with and without social distancing.

identifies areas of 100 by 100 meters with at least 20 SIM cards, by using approximate location data from the previous day [15].

Conventional crowd monitoring often focuses on increasing footfall and activity public places such as high streets [24], however, due to the recent outbreak, the focus has shifted towards protecting city dwellers by monitoring compliance with social distancing practices and detecting "hotspots" of overcrowding activities. Another project [32] attempted to predict the maximum capacity of a public space when following social distancing and dynamically plotting the distribution of crowds. Thermal and laser ranging sensors are utilised in Singapore to maintain and enforce social distancing in some venues [29]. To tackle the overcrowding issue in city spaces, manual crowd counting and social distancing applications [14] have seen significant usage in an attempt to prevent the spread of COVID-19 through Contact Tracing systems. However, while these systems are effective to alert individuals who have been in contact with a new positive case of COVID-19 in the last days, such alert takes place when the infection may have already happened. Thus, there is a need for an automated social distancing detection approaches to allow for a quicker and more effective response. Traditional footfall counting systems such as cameras suffer from issues related to area coverage, cost, and lack of information about people's movement. With the recent advancement of short-range wireless technologies such as Bluetooth and WIFI, new data sources have emerged 
that enable continuous and effective tracking of crowd behaviour.

In this project, we propose a robust overcrowding detection system called "CrowdTracing" that aims at detecting overcrowding and sending alert notifications of dense crowd clusters based on affordable people counting devices and a dense clustering approach. The proposed IoT system is based on passive 802.11 (WIFI) sensing technique that implements a polygon-shaped WiFi adaptor mesh and collects WIFI probe requests sent from nearby mobile devices.Analysing the respective signal strength and the MAC address of the probes send by a each transmitting node at the different Access Points (APs), allows for the system to accurately locate individuals within the crowd. Then the people count is processed by density clustering algorithm, namely, Density-based Spatial Clustering (DBSCAN) to extract overcrowding clusters from the probe request records. The detection of clusters can then help in notifying venue managers OR "crowd controllers" of a potentially large crowd within the vicinity of the network mesh (e.g. busy city center). Figure 2 shows an illustration of overcrowded clusters without social distancing. The results achieved on 1000 simulated scenarios, with a classification recall of 0.85 .

\section{BACKGROUND}

With the surge in the number of cases for COVID-19, the governments and local authorities have been looking at ways of reopening cities while fending off the pandemic. Early in the outbreak, lockdowns have been imposed which have restricted movement and mobility. Later on other strategies have been adopted to ease of the restrictions such as contact tracing. Contact Tracing either manually [2] or based on contact tracing apps installed on at least \%60 of the populations' phones to automate the process [3]. When systematically applied, contact tracing will break the chains of transmissions and is thus an essential public health tool for controlling outbreaks. However, there is a need for more robust and real-time approaches to monitor and disperse large gatherings in city settings. One of the latest studies investigated the direct and indirect impact of crowd density on the current COVID-19 pandemic using structural equation modelling. The researchers found that large metropolitan population areas were one of the most significant predictors of infection rates [12]. With the rapid expansion of technologies for smart cities applications, new tools and data sources become available for monitoring and modelling crowd behaviour. Computer vision is a classic method of crowd dynamic monitoring, using camera networks (such as CCTV) or custom-built systems. Researchers have developed single-image crowd counting using Deep Learning approaches [22], and texture analysis [27] to monitor and extract crowd counts and flow. However, camera-based crowd monitoring solutions are limited through accuracy, occlusion, scaling and positioning requirements. Alternatively, Global Positioning System (GPS) is another potential crowd counting technique which enables mobile devices to be localised and traced. A participatory GPS location application [5] was produced for assisting in the management of crowd mobility during large scale events, the application attracted 28,000 users which contributed to 25 million individual location updates. Whilst the research [5] required an app download, data was aggregated to assist in the safe planning of future events. [5] highlights a significant disadvantage of GPS for crowd counting purposes as it requires an application to be installed on the tracked users mobile. In addition to this, the accuracy of GPS technology is highly dependent on environmental factors and the number of GPS satellites in range, making the technology impractical for indoor environments [16]. Alternatively, Wi-Fi(IEEE802.11) and Bluetooth Low Energy (BLE) packet scanning can be utilised to detect other devices in the vicinity, thus enabling crowd monitoring [18]. They contain information about the sending devices, such as their Media Access Control (MAC) addresses and the transmitting frame was sent on, whilst the Receiver Signal Strength Index (RSSI) is calculated using the signal strength of the probe at the AP (receiving) node. Some mobile devices are capable of broadcasting such frames as often as 2000 times an hour [9], and therefore through intersecting these frames, a device for counting nearby nodes can be produced. MAC addresses are often randomised in order to protect the identity of the sender, however,it is still possible to draw meaningful information regarding collective crowd behaviour. Given the above intercepting capabilities, several projects have investigated the use of WiFi antennas for crowdedness estimation and people count applications. For instance, the work in [21] investigates bus occupancy through the combination of probe request data and an estimation algorithm based on the RSSIs of the intercepted probes and the variability of MAC addresses across time. Recent research has explored [25] footfall changes between weekdays and weekends across New York City and personal routing by the implementation of $54 \mathrm{WiFi}$ access points throughout a week. [24] adopts class-intervals and a graph-based technique for estimating the real-time footfall of high-streets from $\mathrm{WiFi}$ probe requests. Similarly to [6] proposed a threshold-based approach to overcome RSSI fluctuations with a device-free human detection and tracking system. Their proposed system entails of two major functions:"a wireless communication system and a human detection and tracking system".RSSI measurements are filtered beforehand by the use of a weighted moving average. Compared with WiFi, a Bluetooth (BT) based detection approach could be more accurate due to the smaller transmitting and receiving range. However, since it is more likely for a mobile device to have its WiFi switched on than BT, WIFI has potentially much higher detection rate.

\section{METHODS}

In this study, a multi-fold system, namely CrowdTracing, is proposed as a mean of detecting overcrowding and social distancing based on the probe intercepted by ubiquitous $\mathrm{WiFi}$ APs. The main elements of the system are described as follows, (i) development of WIFI Probes (Figure 3 shows the CrowdTracing Probe including a micro-controller, WIFI $\&$ BLe modules and small battery, (ii)automatic partitioning 
of a large space (e.g.neighbourhoods or cities) into smaller areas (e.g.shopping malls or supermarkets), sing footfall count and Self-organising maps (SOMs), to identify overcrowded areas in which social distancing rules are not followed, (iii)localisation through probe intersection and trilateration by the use of a network of WiFi APs, (iv) a density-based clustering algorithm with a pre-defined social distancing and minimal cluster points to detect whether social distancing rules are followed in a specific area, and (v) real-time alert system. The use of SOM in the first stage has the following two objectives: first, to provide a topological view of the data partitioning, and second, to aid the strategical setup of WiFi APs by taking into account the density of the partitions. The user localisation and density-based clustering phase help in discovering clusters of arbitrary shape and identifying groups of people among which social distancing is not being followed. The architecture of CrowdTracing can be seen in Fig. 2.

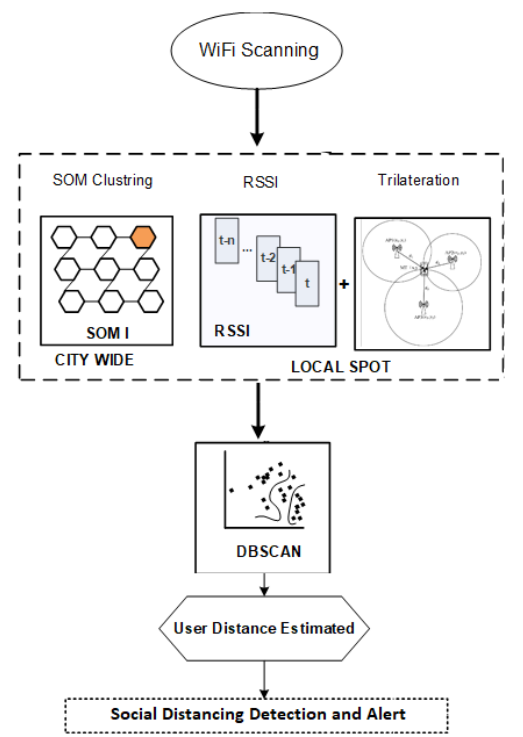

Fig. 2: Schematic model of CrowdTracing framework.

\section{A. People Count System}

We have developed multiple WIFI based people counting probes using various prototypes boards (including ESP32, Arduino Nano and Raspberry Pi 4) attached to PowerBoost $1000 \mathrm{Cs}$ given to make these IoT devices entirely portable, which helps in performing tracking anywhere.The WiFi sniffers packets have been encrypted and directly transmitted to a remote database using local WIFI connection (the boards supports both WIFI and BT).

\section{B. Spatial Clustering}

Clustering is an unsupervised segmenting task which given a feature space, divides the population of data points or training set into different groups such that data points in the same groups are more similar to each other than to those in other groups. There are many clustering techniques including:

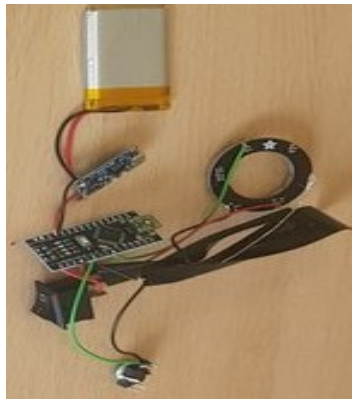

Fig. 3: CrowdTracing WIFI Probe.

1) Centroid-based clustering: such as is iterative in which the similarity between data points is computed as their respective distance to the centroids of a user-defined number of clusters $k$, where those centroids are iteratively re-located to minimise the distance, normally Euclidean, between them and the points associated to their respective clusters. Given the need for the definition of $k$ prior to the training phase, an a priori knowledge upon the structure of the training data points is assumed. One of the popular Centroid-based clustering algorithms is $\mathrm{K}$-means [13].

2) Distribution clustering: is based on distribution, assume the data is composed of probability distributions, with the Gaussian distribution being the most widely used. In distribution clustering, as the distance from a data point to the center of a distribution increases, the probability of that point belonging to that distribution-based cluster decreases. This type of clustering includes, among others, Gaussian Mixture Models (GMMs) [23].

3) Hierarchical clustering: operates either according to a top-down (divisive) or a bottom-up (agglomerative) approach. On the one hand, the top-down approach starts with every data point of a given set in the same unique cluster and make consecutive divisions based on the similarity or dissimilarity between the different points, which ultimately leads to smaller clusters [17]. On the other hand. in the bottom-up approach, every data point is considered a unique cluster which is a posteriori merged with other clusters regarding their similarity. Both the bottom-up and the top-down approaches are stopped when a given stopping criterion is met.

4) Density-based clustering: is based on the identification of areas where data points are concentrated, while considering less concentrated areas and sparse data points as noise. In this context, the distance between the points in a cluster and the density (minimum number of points for a group of data points to be considered as a cluster) can be adjusted according to the requirements of the application. Within density-based clustering techniques, the Density-based spatial clustering (DBSCAN) [8] is the most widely used.

Given the characteristics of the above types of clustering, density-based clustering provides a convenient platform for 
its application on the detection of the maintenance of social distancing rules. This is based on two main factors. First, density-based clustering can detect clusters of arbitrary shapes, that is, people in close proximity can be detected regardless of their respective position to each other. Second, the parameter $(\epsilon)$ with regards to the minimum distance between two points to be considered in the same cluster, can be adjusted with regards to the application. Thus, $\epsilon$ can be continuously adjusted according to the latest instructions given by the corresponding government bodies or medical institutions. Spatial clustering is the process of identifying agglomerations in spatial data, such as those produced by moving objects (e.g., vehicles and pedestrians). Overview Given the application of the proposed system, which is that of monitoring people gatherings, the focus was set to density clustering. Therefore we adopt a state-of-the-art density clustering algorithm DBSCAN which employs two main parameters: the distance $\epsilon$ and the minimum number of points MinPts that must be within a radius $\epsilon$ for these points to be considered as a cluster. The two parameters are considered, an estimate of the point density of the clusters. There are other variations of DBSCAN clustering, for examples, OPTICS long time is explained by the fact that the algorithm is an amelioration of DBSCAN that does not need the specification of a maximum distance $\epsilon$ but adds some overhead. In addition, DeLiClu have a long execution time (nearly $400 \mathrm{~ms}$ ) and this is due to the indexing phase that takes nearly $25 \%$ of the total time. Because of the low variation of density between groups, OPTICS and DeLiClu attempt to group together distant point by considering their density as low. With it's fixed minimal distance $\epsilon$ DBSCAN can limit the density and therefor avoid the problem that OPTICS and DeLiClu face.

\section{Density Mapping for City Zoning}

Before performing clustering via density detection, we identify crowd dense areas within a city where people are likely to gather are identified and mapped. For this we utilise SelfOrganizing Map (SOM), which is a type of artificial neural network (ANN) SOM is trained using unsupervised learning to produce a low-dimensional (typically two-dimensional), discretized representation of the input space of the training samples, called a map [20]. In addition, SOM is a well established non-linear mapping tool, and it has many beneficial properties, such as tolerance for incomplete and small data set [10], however, few issues need to be tackled to get efficient results. [28] proposed to train the SOM in two phases: ordering phase and tuning phase, to quickly scan a large area and to produce a well organised and tightly coupled map. It is also suggested to employ heuristics measures to evaluate the efficiency of the trained SOM by measuring and comparing the quantization error (QE) and the topographic error (TE), eventually aiming for a low TE and QE.

The proposed SOM clustering model consists of one unit with a $p$ processing neurons each made of input nodes and a competitive layer or output layer of neurons where the groupings are taking place. Usually, SOM learn to classify

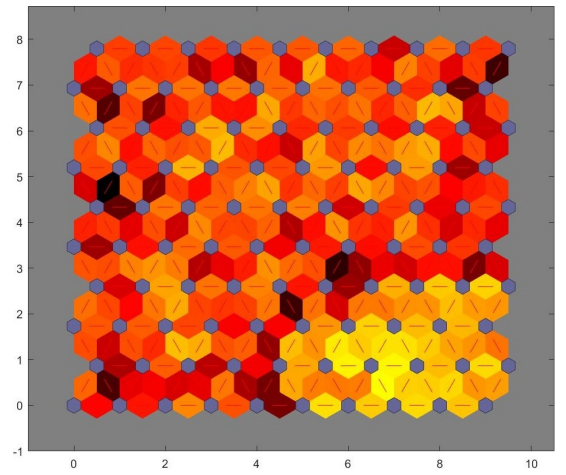

Fig. 4: SOM neuron weight positions of the selected APs data clustering map.

input nodes according to how they are grouped in the input space, therefore, it could recognise similar distance according to their crowed score index and it would organise those with similar scores. Thus, SOMs learn both the distribution and topology of the input nodes they are trained on.

To demonstrate its relevance to the overcrowding clustering problem, we utilised Open Access data from people count Access Points setup by Newcastle Urban Observatory project [1](located in the City Center of Newcastle). The people count data crawled from four APs between 15th of May and 15th of June (2020) for 24 hours throughput. Since there is no strict scientific method to determine the right size for the SOM map, thus, different values are experimented till satisfied with the results using a 10 by 10 layer of neurons to produce $p=100$ different meaningful classification for crowed clusters. In Figure 4, each neuron is represented by a grey dot at the location of its two weights. The figure shows the map after training when the map started to self-organise so that each neuron organises different groups of the input space, and related neurons get connected to the nearby neighbouring group. To visualise the example results obtained by SOM in a user-friendly fashion, large clusters of people are counted in hive shaped map where over crowding (more than 6 people) are given different sized hive cell as shown in Fig 4.

\section{Social Distancing Tracking}

This section presents the proposed unobtrusive system to detect whether social distancing rules are being followed in crowd dense areas.

1) Data Generation: To validate the social distancing detection algorithm proposed, a synthetic data set was generated as follows. Let A be a 30x30m squared area with an access point located at each of the four corners $((0,0),(0,30),(30,0),(30,30))$ and $n_{\text {simulations }}=1000$ be the number of simulated scenarios. In each simulated scenario a total of $N$ users, where $10 \leq N \leq 40$, are created at random coordinates $\left(X_{\text {real }}, Y_{\text {real }}\right)$ within A. The distances from each user $\mathrm{n}$ to each of the APs are calculated as their respective Euclidean distance. Such distances are then converted to their 


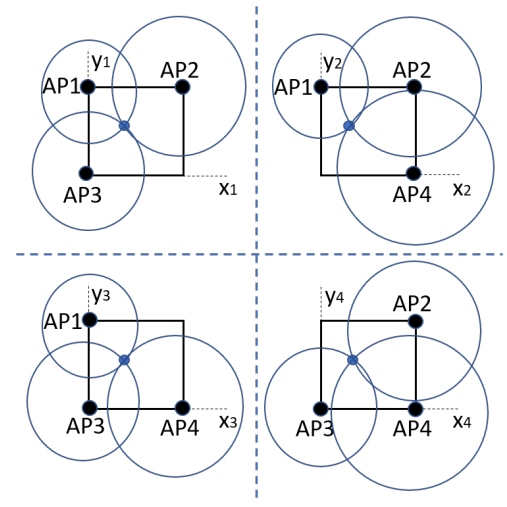

Fig. 5: Trilateration averaging using three AP at the time.

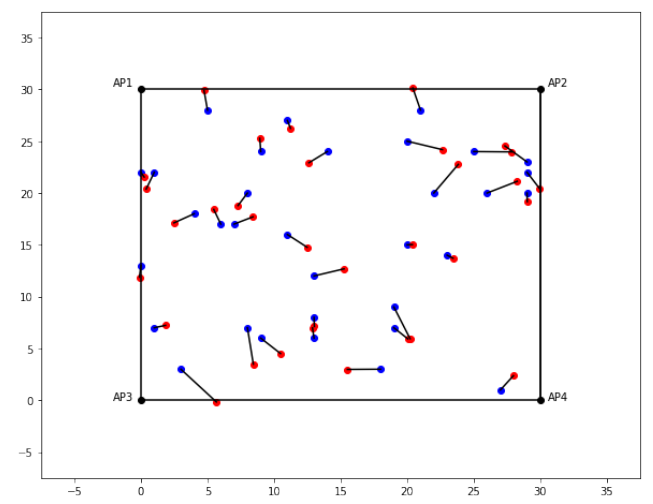

Fig. 6: Example of a simulated scenario. The blue dots represent real users, and red dots represent the added-noise. The black lines connecting the dots represent the resultant coordinates after distance-RSSI-distance transformation.

respective hypothetical RSSI values by:

$$
R S S I=-M_{p}-2 E_{f} \log _{10} D
$$

where $M_{p}=-40$ is the expected RSSI at 1 meter distance from an access point and $E_{f}=2$ is a constant environmental factor.

Random noise from the normal distribution $(\mu=0, \sigma=1)$ added to each of the estimated RSSI values and then these are reconverted to distances using Equation 1. Through the use of trilateration averaging, the new coordinates $\left(X_{\text {noisy }}, Y_{\text {noisy }}\right)$ of each user $\mathrm{n}$ are estimated as the average coordinates given by four trilateration processes using three access points at the time (AP1,AP2,AP3), (AP1,AP2,AP4), (AP1,AP3,AP4), (AP2,AP3,AP4) (see Fig. 5). The final coordinates are thus given by:

$$
\left(\frac{x_{1}+x_{2}+x_{3}+x_{4}}{4}\right),\left(\frac{y_{1}+y_{2}+y_{3}+y_{4}}{4}\right)
$$

For illustration purposes, the real and the resultant noisy coordinates of the different $\mathrm{N}$ users in a simulated scenario are shown in Fig. 6.

2) Density Clustering: Provided the data presented in Section III-D1, the system proposed aims at detecting whether

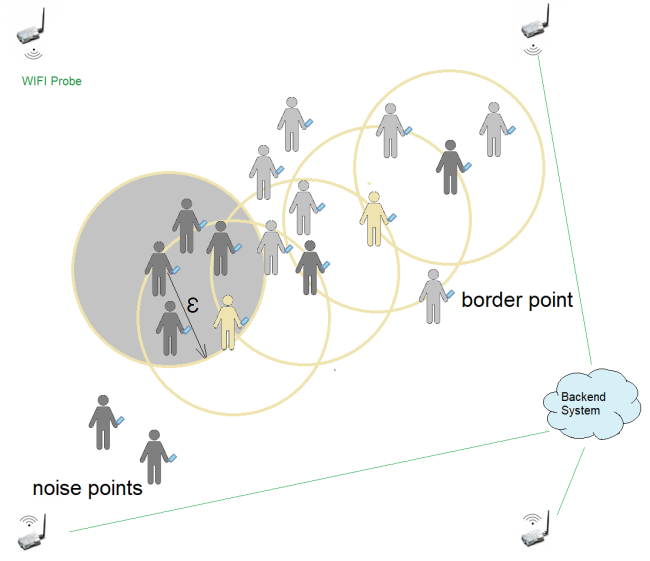

Fig. 7: Example of density-based spatial clustering of people.

social distancing rules are kept in each independent scenario based on clustering algorithms. As mentioned in Section III-B, DBSCAN is favour over other clustering algorithms due to its robustness and minimum overhead. In addition, DBSCAN ability to configure a distance and minipoints (for minimum number of people) and minimum distance between objects makes it a perfect fit for detecting overcrowding when people are not maintaining the recommended social distance among themselves in open spaces. The $\epsilon$ neighborhoods of a point $\mathrm{x}$ is the set of points in the data set whose distance to $\mathrm{x}$ is less than $\epsilon ; N_{\epsilon}(\mathrm{x})=\{\mathrm{u} \in X \mid=d(u, x)<\epsilon\}$. DBSCAN recursively visits each object $\mathrm{u} \in \mathrm{N}_{\epsilon}(\mathrm{x})$ in the neighbourhood of a core u object, in order to check if $\mathrm{u}$ is also a core object, i.e. if it also has minPts neighbours. By such, the cluster is recursively expanded until no further objects are added to the cluster, i.e., all objects checked in the recursion step are border objects or have been previously visited.

Let $\Gamma$ be the minimum number of users within the radius $\epsilon$ for the neighbourhood to be considered as not keeping social distancing rules. Using the real coordinates $\left(X_{\text {real }}, Y_{\text {real }}\right)$ from each of the users, $\epsilon=2$ meters, and $\Gamma=4$, it determines if these users are maintaining social distancing. The intuition behind the use of $\Gamma=4$ is to avoid system alerts when triggered by small group of people (bubble) from the same household. A scenario is then labelled as ' 1 ' if the number of clusters given by the DBSCAN is greater than $1\left(n_{\text {clusters }}>\right.$ $1)$, and 0 otherwise. The noisy coordinates $\left(X_{\text {noisy }}, Y_{\text {noisy }}\right)$, $\epsilon$ is used as a hyper-parameter of the clustering problem to maximise the matching between the noisy scenarios and the real scenarios when detecting social distancing. Figure 7 shows an example of crowd detection using density clustering in city setting.

\section{Clustering Results}

As mentioned in the previous section, the performance of the system is studied as a function of the radius of neighbourhood $\epsilon$. The intuition behind this, is to account for the undesired noise typically present in the RSSI of radio 


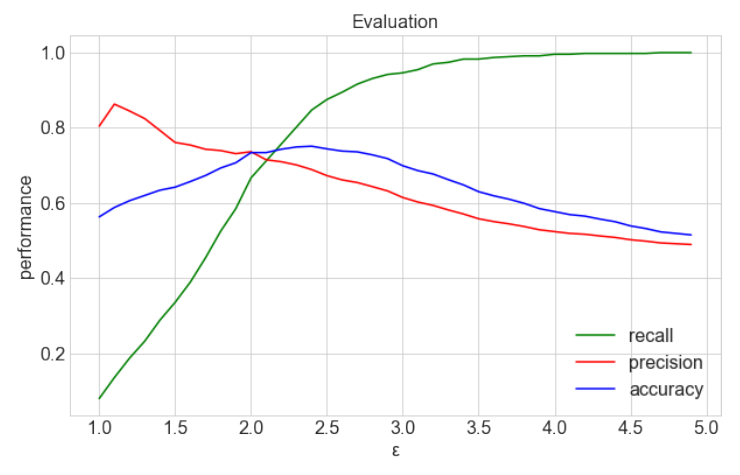

Fig. 8: System accuracy, precision and recall as a function of the radius of neighbourhood $\epsilon$.

frequency (RF) signals. The results obtained are shown in Fig. 8.

While it is crucial to account for the undesired noise present in the RSSI allowing for certain expansion of the neighbourhood radius as compared to that used with real coordinates and to identify the maximum number of scenarios where social distancing rules are not followed, it also important not to generate many false alarms which can unnecessarily disturb policing institutions. Thus, a fair balance should be kept between the recall and precision measures of the system. Certainly, increasing the radius of neighbourhood $\epsilon$, will lead to higher recall values, however, such increase can lead to a excessively high number of false positives. Given this, the classification accuracy is considered as the reference measure to report the optimal results achieved by the system. The maximum accuracy (0.75) is achieved when $\epsilon=2.40$. This leads to a precision of 0.69 and a recall of 0.85 . The corresponding confusion matrix is shown in Fig. 9. By analysing the confusion matrix it can be stated that out of the 1000 simulated scenarios, 357 were correctly classified as having groups of 4 or more people not respecting the social distancing rules, 394 were correctly classified as scenarios where social distancing rules were being followed, 178 were mistakenly predicted as scenarios where social distancing rules were respected, and 71 were mistakenly predicted as scenarios where social distancing rules were being respected.

\section{Opportunities \& Challenges}

The scanning capabilities of short-range wireless communications APs such as BLE and WiFi present an opportunity to exploit the RF signals corresponding to the probes sent by surrounding devices as a means of detecting crowded areas and ensure social distancing rules in place are followed by the public. By leveraging the respective RSSI of the probe requests sent by the surrounding devices at the different APs, it is plausible to have an estimate of the respective distance of each device with the each AP. This information can be exploited as posteriori estimation of the location of each device using the principle of "Trilateration". In contrast to traditional contact tracing approaches, the system proposed

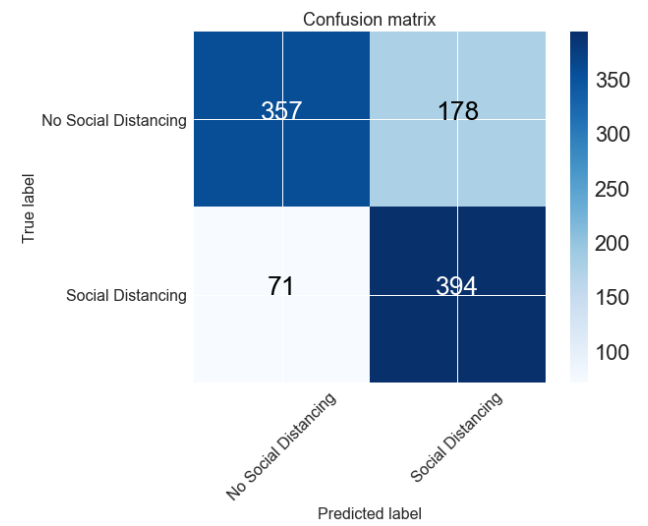

Fig. 9: Confusion matrix of the proposed crowd detection system.

in this paper do not require the installation of mobile apps on users phones while identify overcrowding rather than just one person's close contacts. While the system proposed is not presented as an alternative to contact tracing, we believe its adoption could well complement contact tracing applications in the fight against the spread of viral diseases such as COVID19. Building a system to report the maintenance of social distancing rules in busy areas around the city is challenging, but essential to keep new infection cases at lower levels. In this section, the limitations and challenges of building the proposed system and of its widespread adoption are discussed. The data collected for detecting overcrowding is anonymous and does not reveal any personal information. On the otherhands, not all individuals have mobile phones and some people who have phones might switch off WIFI. Such devices are visible to the network when they perform SSID scans using a randomised MAC address. Ignoring them can yield to undercounting. However, under-counting will not trigger action, and comparing to the number of people with mobile phones with WIFI switched on, we still can reasonably approximate the number of people in each cluster. In addition, we plan to explore the possibility of scanning for nearby BLE which are also available on on most wearables. However, unassociated devices such as laptops, printers or other products may be recognised by the system. To avoid including such devices in the total count, MAC address filtering should take place to only include recognised mobile phone manufacturers. The use of BLE beacons and video cameras to complement our approach will be considered in future work.The Received Signal Strength Indicator (RSSI) is a ratio-based measure that enables devices to evaluate the intensity at which Radio Frequency signals arrive at the location of the receiving node [7]. Although RSSI is becoming increasingly popular for localisation and distance estimation, there are challenges that have to be accounted for when working with such a value. A significant challenge to the reliability of RSSI values is the surrounding environment such as how RF signals are affected by the multi-path effect caused by the walls, ceilings and floors. Many influential factors might impact the quality of 
signal propagation such as humidity and temperature [11] and the presence of human beings around the receiver due to the absorption of molecular resonance [31]. To mitigate this issue, the number of APs need to be increased which could improve the accuracy of triangulation and fingerprinting techniques [30] to few centimeters accuracy [19].

\section{CONCLUSiON}

COVID-19 outbreak is unprecedented and has disrupted lives of millions of people across the globe. This pandemic has opened several research challenges and opportunities that cities must address by introducing place-based interventions to prevent further spread of the virus. The proposed architecture and approach discussed in the article can be used to effectively and timely manage social distancing in cities at high granularity levels by exploiting logs gathered by WiFi APs deployed in urban spaces as The initial results achieved by the proposed CrowdTracing system is promising, which potentially can help in the identification of highly-dense crowded places within a city by combining density mapping techniques such as SOM with the employment of ubiquitous Wi-Fi APs. These APs intercept and analyse the rate of the probe requests sent by the adjacent WiFi-enabled devices. In addition, the results achieved by the system aiming at identifying whether social distancing rules are followed within specific areas, with a classification recall of 0.85 , suggest those same APs can be employed to monitor social distancing in areas of interest.

\section{REFERENCES}

[1] Access points data, newcastle urban observatory, accessed online on may 2021. URL: https://newcastle.urbanobservatory.ac.uk/.

[2] Nhs test and trace: if you've been in contact with a person who has coronavirus, Jun 2020. URL: https://www.nhs.uk/conditions/coronaviruscovid-19/testing-and-tracing/nhs-test-and-trace-if-youve-been-incontact-with-a-person-who-has-coronavirus/.

[3] Ali Alkhatib. We need to talk about digital contact tracing. Personal weblog. May, 1, 2020.

[4] Dario Ortega Anderez, Eiman Kanjo, Ganna Pogrebna, Omprakash Kaiwartya, Shane D. Johnson, and John Alan Hunt. A covid-19-based modified epidemiological model and technological approaches to help vulnerable individuals emerge from the lockdown in the uk. Sensors, 20(17), 2020. URL: https://www.mdpi.com/1424-8220/20/17/4967, https://doi.org/10.3390/s20174967 doi:10.3390/s20174967.

[5] Ulf Blanke, Gerhard Tröster, Tobias Franke, and Paul Lukowicz. Capturing crowd dynamics at large scale events using participatory gpslocalization. In 2014 IEEE Ninth International Conference on Intelligent Sensors, Sensor Networks and Information Processing (ISSNIP), pages 1-7. IEEE, 2014.

[6] Apidet Booranawong, Nattha Jindapetch, and Hiroshi Saito. A system for detection and tracking of human movements using rssi signals. IEEE sensors journal, 18(6):2531-2544, 2018.

[7] Yogita Chapre, Prasant Mohapatra, Sanjay Jha, and Aruna Seneviratne. Received signal strength indicator and its analysis in a typical wlan system (short paper). In 38th Annual IEEE Conference on Local Computer Networks, pages 304-307. IEEE, 2013.

[8] Martin Ester, Hans-Peter Kriegel, Jörg Sander, Xiaowei Xu, et al. A density-based algorithm for discovering clusters in large spatial databases with noise. In $K d d$, volume 96, pages 226-231, 1996.

[9] Julien Freudiger. How talkative is your mobile device? an experimental study of wi-fi probe requests. In Proceedings of the 8th ACM Conference on Security \& Privacy in Wireless and Mobile Networks, pages 1-6, 2015.

[10] M. H. Ghaseminezhad and A. Karami. A novel self-organizing map (som) neural network for discrete groups of data clustering. Applied Soft Computing, 11(6):3771-3778, 062011.
[11] Amir Guidara, Ghofrane Fersi, Faouzi Derbel, and Maher Ben Jemaa. Impacts of temperature and humidity variations on rssi in indoor wireless sensor networks. Procedia Computer Science, 126:1072-1081, 2018.

[12] Shima Hamidi, Sadegh Sabouri, and Reid Ewing. Does density aggravate the covid-19 pandemic? early findings and lessons for planners. Journal of the American Planning Association, pages 1-15, 2020.

[13] John A Hartigan and Manchek A Wong. Algorithm as 136: A k-means clustering algorithm. Journal of the royal statistical society. series $c$ (applied statistics), 28(1):100-108, 1979.

[14] Enrique Hernández-Orallo, Pietro Manzoni, Carlos T Calafate, and JuanCarlos Cano. Evaluating how smartphone contact tracing technology can reduce the spread of infectious diseases: the case of covid-19. IEEE Access, 2020.

[15] Sepp Huber. Swisscom analyses in the fight against coronavirus, Mar 2020. URL: https://www.swisscom.ch/en/about/news/2020/03/27swisscom-coronavirus.html.

[16] Soyoung Hwang and Donghui Yu. Gps localization improvement of smartphones using built-in sensors. International Journal of Smart Home, 6(3):1-8, 2012.

[17] Stephen C Johnson. Hierarchical clustering schemes. Psychometrika, 32(3):241-254, 1967.

[18] Eiman Kanjo. Tools and architectural support for mobile phones based crowd control systems. Network Protocols and Algorithms,4 (3), 4-14.

[19] Jaewon Kim and Dongsoo Han. Passive wifi fingerprinting method. In 2018 International Conference on Indoor Positioning and Indoor Navigation (IPIN), pages 1-8. IEEE, 2018.

[20] Yeuvo Kohonen. The self-organizing map. Proceedings of IEEE, 78(9): 1464 - 1480, 1990.

[21] Lars Mikkelsen, Radoslav Buchakchiev, Tatiana Madsen, and Hans Peter Schwefel. Public transport occupancy estimation using wlan probing. In 2016 8th International Workshop on Resilient Networks Design and Modeling (RNDM), pages 302-308. IEEE, 2016.

[22] Vy Nguyen and Thanh Duc Ngo. Single-image crowd counting: a comparative survey on deep learning-based approaches. International Journal of Multimedia Information Retrieval, pages 1-18, 2019.

[23] Douglas A Reynolds. Gaussian mixture models. Encyclopedia of biometrics, 741, 2009.

[24] Balamurugan Soundararaj, James Cheshire, and Paul Longley. Estimating real-time high-street footfall from wi-fi probe requests. International Journal of Geographical Information Science, 34(2):325-343, 2020.

[25] Martin W Traunmueller, Nicholas Johnson, Awais Malik, and Constantine E Kontokosta. Digital footprints: Using wifi probe and locational data to analyze human mobility trajectories in cities. Computers, Environment and Urban Systems, 72:4-12, 2018.

[26] Kieran Woodward, Eiman Kanjo, Dario Ortega Anderez, Amna Anwar, Thomas Johnson, and John Hunt. Digitalppe: Low cost wearable that acts as a social distancingreminder and contact tracer: Poster abstract. In Proceedings of the 18th Conference on Embedded Networked Sensor Systems, SenSys '20, page 758-759, New York, NY, USA, 2020. Association for Computing Machinery. https://doi.org/10.1145/3384419.3430600 doi:10.1145/3384419.3430600.

[27] Xinyu Wu, Guoyuan Liang, Ka Keung Lee, and Yangsheng Xu. Crowd density estimation using texture analysis and learning. In 2006 IEEE international conference on robotics and biomimetics, pages 214-219. IEEE, 2006.

[28] Vineet Yadav and Dipti Srinivasan. A som-based hybrid linear-neural model for short-term load forecasting. Neurocomputing, 74(17):2874 2885, 2011.

[29] Elaine Yau. Singapore company's tech that monitors social distancing, Jun 2020. URL: https://www.scmp.com/lifestyle/healthwellness/article/3089201/singaporean-companys-new-tech-policesocial-distancing.

[30] Simon Yiu, Marzieh Dashti, Holger Claussen, and Fernando Perez-Cruz. Wireless rssi fingerprinting localization. Signal Processing, 131:235244, 2017.

[31] Moustafa Youssef, Matthew Mah, and Ashok Agrawala. Challenges: device-free passive localization for wireless environments. In Proceedings of the 13th annual ACM international conference on Mobile computing and networking, pages 222-229, 2007.

[32] Álvaro González García, James L. Martín R., and Alessio Caciagli. Crowd control in plazas constrained to social distancing, 2020. http://arxiv.org/abs/2005.07038 arXiv:2005.07038. 